\title{
Pancreaticoduodenectomy for advanced gastric cancer
}

\author{
Makoto Saka, Satvinder S. Mudan, Hitoshi Katai, Takeshi Sano, Mitsuru Sasako, and Keitchi Maruyama \\ Department of Surgical Oncology, National Cancer Center Hospital, 5-1-1 Tsukiji, Chuo-ku, Tokyo 104-0045, Japan
}

\begin{abstract}
Background. Although pancreaticoduodenectomy has been rarely performed for gastric caner because of frequent morbidity and mortality, some favorable results after this procedure have been reported recently. Our objective was to present our data that might aid in the selection of patients to undergo this procedure.

Methods. Between 1970 and 2001, 23 patients who had pancreaticoduodenectomy for gastric cancer with tumor invading the pancreatic head were identified, and they were the subjects of this study. Clinical, operative, and pathological data, and morbidity and mortality rates were collected and analyzed. Survival outcome was also calculated and analyzed. Results. Five patients underwent this procedure for disease in the gastric remnant, 18 undergoing the procedure for primary tumors. Median operating time was $8 \mathrm{~h}$ (range, 6-13h), and median blood loss was $1600 \mathrm{ml}$ (range, $700-16000 \mathrm{ml}$ ). Regarding extent of gastrectomy, all patients with primary cancer $(n=18)$ underwent a distal gastrectomy and patients with disease in the gastric remnant $(n=5)$ underwent a completion gastrectomy. Incurable factors, including paraaortic lymph node metastasis, positive lavage cytology, or peritoneal dissemination were found in 8 patients. The postoperative morbidity rate was $73.9 \%$; however, operation-related death was zero. The overall 5-year survival rate was $34.3 \%$. The 5-year survival rate of the 8 patients with incurable factors was $0 \%$, while that of the 15 patients without incurable factors was 47.4\%.

Conclusion. If an $\mathbf{R} 0$ resection can be achieved by pancreaticoduodenectomy, this procedure should be performed for patients with tumor invading the pancreatic head. Patients with incurable factors should not be considered for pancreaticoduodenectomy.
\end{abstract}

Key words Gastric cancer · Pancreaticoduodenectomy · Combined resection of adjacent organs

Offprint requests to: $\mathrm{H}$. Katai

Received: April 8, 2004 / Accepted: September 1, 2004

\section{Introduction}

Complete removal of all evaluable disease, i.e., R0 resection, is vital to a successful outcome in gastric cancer treatment. Extended surgery is occasionally required for advanced gastric cancer with infiltration of adjacent organs to achieve complete tumor clearance. For locally advanced gastric cancer with infiltration of the pancreatic head or duodenum, pancreaticoduodenectomy $(\mathrm{PD})$ is required. However, this procedure has been rarely performed because of substantial morbidity and mortality [1]. Prior to the 1990s, few reports regarding PD for gastric cancer had been published [2]. Only Kishimoto et al. [3] and Scott et al. [4] referred to a long survivor after this procedure in their reports about gastrectomy with combined resection. Recently, with current advances in operative techniques and in nutritional support, some favorable results of the patients undergoing this procedure have been reported [5-7]. However, only a few reports with a large number of cases have been published so far. In the current study, we present our data that might aid in the selection of patients to consider who should undergo this procedure.

\section{Subjects and methods}

A retrospective review of our prospective database, spanning from 1970 to 2001 and containing 9349 patients, identified 195 (2.1\%) who had locally advanced cancer with macroscopically suspected infiltration of the pancreatic head. We included patients with pancreatic head invasion from metastatic lymph nodes, and excluded type 4, linitis plastica cancer. Of the 195 patients identified, 23 underwent PD with presumed curative intent, and they were the subjects of this study.

In these 23 patients, clinical data, including age, sex, symptoms, and primary tumor or tumor in the gastric remnant, were collected and analyzed, using the appro- 
Table 1. Patients undergoing pancreaticoduodenectomy

\begin{tabular}{|c|c|c|c|c|c|c|c|c|c|c|c|c|}
\hline & & Disease & Stage & $\mathrm{pT}$ & $\mathrm{pN}$ & $\mathrm{P}$ & CY & $\begin{array}{l}\text { Adjuvant } \\
\text { Chemo. }\end{array}$ & $\begin{array}{l}\text { Combined } \\
\text { resection }\end{array}$ & Recurrence & $\begin{array}{c}\text { FUT } \\
\text { (months) }\end{array}$ & Status \\
\hline 1 & $63 / \mathrm{F}$ & Primary & IV & 4 & 1 & 0 & 0 & - & Liver & $\mathrm{N}$ & 13 & DOD \\
\hline 2 & $42 / \mathrm{M}$ & Primary & IIIB & 3 & 1 & 0 & ND & - & & - & 157 & DOC \\
\hline 3 & $64 / \mathrm{M}$ & Primary & IIIB & 2 & 2 & 0 & 0 & - & & - & 182 & NED \\
\hline 4 & $67 / \mathrm{M}$ & Primary & IV & 3 & 2 & 0 & ND & - & & - & 87 & DOC \\
\hline 5 & $76 / \mathrm{M}$ & Primary & IV & 4 & 3 & 0 & 0 & - & Colon & Unclear & 4 & DOD \\
\hline 6 & $67 / \mathrm{M}$ & Primary & IIIB & 4 & 0 & 0 & 0 & + & & - & 26 & DOC \\
\hline 7 & $65 / \mathrm{M}$ & Primary & IV & 4 & 3 & 0 & 1 & + & & $\mathrm{N}$ & 6 & DOD \\
\hline 8 & $74 / \mathrm{F}$ & Primary & IV & 2 & 3 & 0 & 0 & - & Colon & $\mathrm{H}$ & 34 & AWD \\
\hline 9 & $70 / \mathrm{M}$ & Primary & IV & 4 & 2 & 0 & 0 & - & Colon & $\mathrm{N}, \mathrm{H}$ & 14 & DOD \\
\hline 10 & $62 / \mathrm{M}$ & Primary & II & 2 & 0 & 0 & 0 & - & Colon & - & 52 & NED \\
\hline 11 & $65 / \mathrm{M}$ & Primary & IV & 4 & 2 & 0 & 0 & - & & $\mathrm{N}$ & 36 & AWD \\
\hline 12 & $65 / \mathrm{F}$ & Primary & IV & 4 & 2 & 0 & 0 & - & & $\mathrm{N}, \mathrm{H}$, spleen & 12 & DOD \\
\hline 13 & $58 / \mathrm{M}$ & Primary & IV & 4 & 3 & 0 & 0 & - & Colon & $\mathrm{N}$ & 6 & DOD \\
\hline 14 & $60 / \mathrm{M}$ & Primary & IIIB & 2 & 2 & 0 & 0 & - & Colon & - & 12 & NED \\
\hline 15 & 64/M & Primary & IV & 4 & 2 & 1 & 1 & - & Colon & Unclear & 19 & DOD \\
\hline 16 & $51 / \mathrm{F}$ & Primary & IIIB & 2 & 2 & 0 & 0 & - & & $\mathrm{H}$ & 11 & DOD \\
\hline 17 & 61/M & Primary & IV & 4 & 1 & 0 & ND & - & & $\mathrm{H}$ & 4 & DOD \\
\hline 18 & $70 / \mathrm{M}$ & Primary & IV & 4 & 3 & 0 & 1 & - & & $\mathrm{N}$, lung & 4 & DOD \\
\hline 19 & $60 / \mathrm{M}$ & Remnant & IV & 4 & 2 & 1 & 1 & - & & $\mathrm{N}$ & 13 & DOD \\
\hline 20 & $57 / \mathrm{M}$ & Remnant & IV & 4 & 1 & 0 & 0 & - & Liver, colon & $\mathrm{N}, \mathrm{H}$ & 26 & DOD \\
\hline 21 & $64 / F$ & Remnant & IIIB & 4 & 0 & 0 & 0 & - & & $\mathrm{N}$ & 64 & DOD \\
\hline 22 & $47 / \mathrm{M}$ & Remnant & IV & 4 & 3 & 0 & 0 & - & & $\mathrm{N}$ & 17 & DOD \\
\hline 23 & $60 / \mathrm{M}$ & Remnant & IIIB & 4 & 0 & 0 & 0 & - & Colon & $\mathrm{P}$ & 4 & AWD \\
\hline
\end{tabular}

Primary, Primary tumor; remnant, tumor of the gastric remnant; P, peritoneal dissemination; CY, lavage cytology; ND, not done; N, lymph node; $\mathrm{H}$, liver; FUT, follow-up time; NED, no evidence of disease; AWD, alive with disease; DOC, dead of other cause; DOD, dead of disease; unclear, site of recurrence unclear

priate nonparametric tests. Operative data, including operating time, blood loss, hospital stay, extent of gastrectomy, extent of lymphadenectomy, and combined resection with $\mathrm{PD}$, were also evaluated. Pathological data, including pT, pN stage, site of tumor, and incurable factors, such as paraaortic lymph node metastasis (pN3), peritoneal dissemination, and positive lavage cytology, were analyzed according to the Japanese classification. Perioperative morbidity and mortality were also investigated.

The survival data of the 195 patients with tumors invading the pancreatic head, including the $23 \mathrm{PD}$ patients, were calculated by the Kaplan-Meier method and analyzed by the log-rank method.

\section{Results}

\section{Demographics}

Of the 195 patients with tumors invading the pancreatic head, $151(77 \%)$ underwent resection, and the remaining 44 underwent only an exploration or a bypass surgery. In 68 patients, an R0 resection was carried out. In 45 patients with $\mathrm{R} 0$ resections, a lesser pancreatic resection (not PD) was performed because of a slight degree of tumor infiltration. The remaining 23 patients $(12 \%)$ underwent PD (Fig. 1).

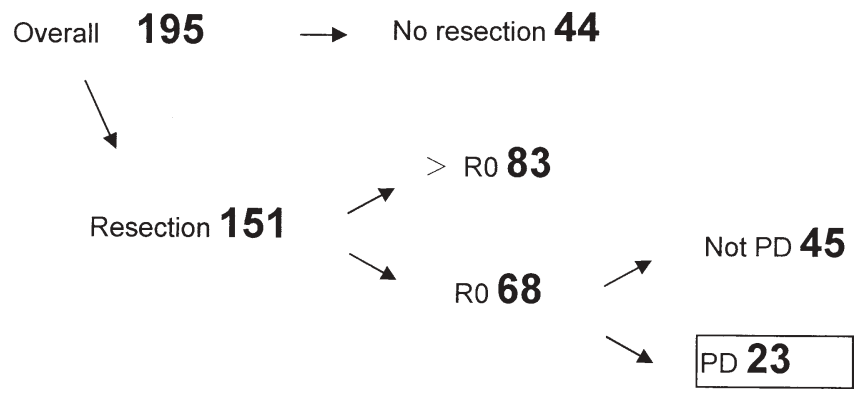

Fig. 1. Patients with tumors invading the pancreatic head. No resection, patients undergoing only exploration or bypass operation. Not PD, patients undergoing R0 resection, but with a lesser pancreatic resection than pancreaticoduodenectomy $(P D)$

In the 23 patients undergoing $\mathrm{PD}$, the median age at the time of resection was 64 years (range, 42-76 years), with a male-to-female ratio of 18:5 (Table 1). Twenty-one patients $(91.3 \%)$ were symptomatic, most commonly with abdominal pain $(n=13)$ and symptoms due to obstruction, including fullness and vomiting $(n=11)$.

Eighteen patients underwent the PD procedure for primary cancer and 5 for gastric remnant cancer following previous Billroth I gastrectomy. Of the 5 patients with gastric remnant cancer, 4 had undergone distal partial gastrectomy for gastric cancer. Two of these 
patients had early cancers, and the other 2 had advanced disease. The disease-free intervals were 1.5 and 6 years for those with advanced cancers and 8 and 10 years in those with early cancers. The fifth patient had had a partial gastrectomy for a benign gastric ulcer 30 years previously.

\section{Operative data}

The median operating time for PD was $8 \mathrm{~h}$ (range, 6-13 h), with a blood loss of $1600 \mathrm{ml}(700-16000 \mathrm{ml})$. The median length of postoperative hospital stay was 37 days (range, 25-92 days). Regarding extent of gastrectomy, patients with primary cancer $(n=18)$ underwent a distal gastrectomy and those with gastric remnant cancer $(n=5)$ underwent a completion gastrectomy. As to extent of lymph node dissection, 14 patients underwent D2 lymphadenectomy and 9 underwent D3. In 9 patients, a combined resection of the colon was performed because of direct infiltration of the mesocolon (Table 1). Two patients underwent a partial hepatectomy because of a direct invasion of the liver. Modified Child's method was selected for a reconstruction for all patients. Two patients received postoperative adjuvant chemotherapy of 5-fluoruracil (5-FU) after surgery.

\section{Pathology}

Resection specimens from all patients revealed adenocarcinoma of gastric origin. In 7 patients, infiltration of the pancreatic head could not be confirmed histopathologically. Regarding site of tumor, 18 primary tumors involved the antrum, and 11 of these tumors extended into the duodenum.

Incurable factors, including $\mathrm{pN} 3$, peritoneal dissemination, and positive lavage cytology were found in eight patients (Table 1). No patient in this series had a visceral metastasis. In 6 patients, pN3 was found. These patients had been considered as negative for $\mathrm{pN} 3$ intraoperatively, but the finding was changed to positive by pathological examination postoperatively. Of these 6 patients, 2 also had positive lavage cytology. Two patients had positive lavage cytology and peritoneal dissemination synchronously; the peritoneal dissemination was a single nodule that was removed easily at operation.

Seventeen patients developed recurrences. The most common recurrence sites were nodal, in 11 patients, followed by liver, in 6 ; peritoneum in 1 ; lung in 1 , spleen in 1 , and unclear, in 2.

\section{Morbidity and mortality}

Postoperative complications were seen in 17 patients (73.9\%; Table 2). Pancreatic fistula was the most
Table 2. Postoperative morbidity

$n$

Postoperative morbidity

$17(73.9 \%)$

Pancreatic fistula

$10(43.5 \%)$

Abdominal abscess

Anastomotic or jejunal stenosis

Cholangitic infection

$3(13.0 \%)$

$3(13.0 \%)$

$3(13.0 \%)$

Anastomotic leakage

$2(8.7 \%)$

Table 3. Survival of patients with tumor invading the pancreatic head

\begin{tabular}{lrcc}
\hline & & $\begin{array}{c}\text { Median } \\
\text { survival } \\
\text { (months) }\end{array}$ & $\begin{array}{c}\text { 5-Year survival } \\
\text { rate } \\
(\%)\end{array}$ \\
\hline Overall & 195 & 10 & 13.6 \\
No resection & 44 & 7 & 0 \\
Resection & 151 & 12 & 17.7 \\
P R0 & 83 & 8 & 7.9 \\
R0 & 68 & 21 & 29.3 \\
$\quad$ Not PD & 45 & 22 & 28.1 \\
$\quad$ PD & 23 & 17 & 34.3 \\
\hline
\end{tabular}

No resection, Patients who underwent only exploration or bypass operation; not PD, patients who underwent $\mathrm{R} 0$ resection but received a lesser pancreatic resection than PD

common. All patients who developed this complication recovered, after receiving drainage and continuous irrigation, using double-lumen drainage tubes. No operation-related death occurred in this series.

Regarding the long-term postoperative morbidity, body weight at 12 months was maintained within $10 \%$ of the preoperative weight in all patients who lived for more than 1 year. Serum albumin levels were not decreased. However, two patients who underwent PD with completion gastrectomy required total parenteral nutrition (TPN) at home, for 1 and 3 years, respectively, after discharge from hospital, because of malnutrition. Postoperative pancreatic endocrine function was adequate in all patients, but three patients required pancreatic exocrine enzyme support postoperatively.

\section{Survival}

In the 195 patients with tumors invading the pancreatic head, the 5-year survival rate was $13.6 \%$. Of these 195 patients, the 68 patients who underwent an $\mathrm{R} 0$ resection showed a better survival outcome, with a 5-year survival of $29.3 \%$. In patients who had R0 resections, there was no significant difference in survival between patients who underwent PD and those not receiving PD (Table 3).

In the 23 PD patients, the median follow-up time was 13 months (range, 4-182 months). The status of the 


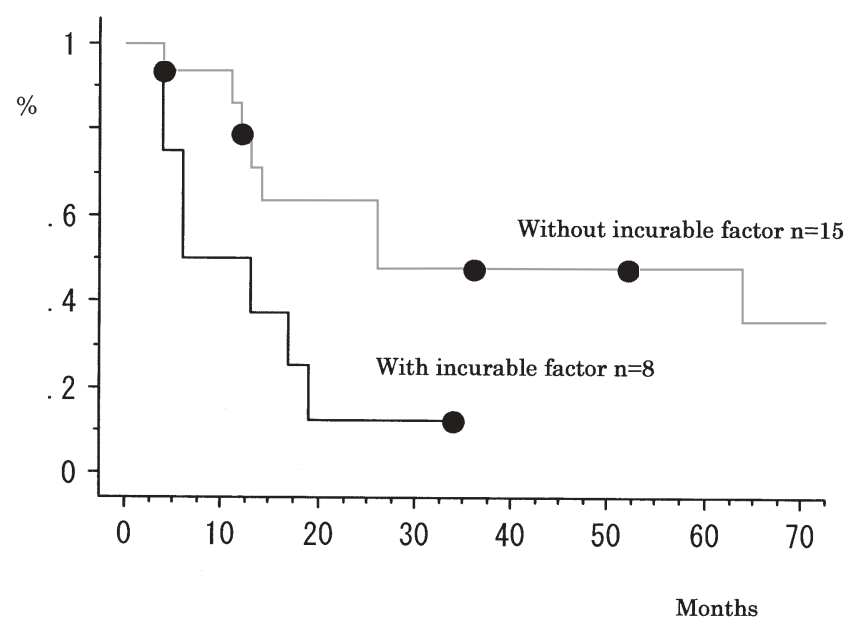

Fig. 2. Survival curves of patients undergoing pancreaticoduodenectomy $(P D)$. The overall 5-year survival rate and the median survival of the 8 patients with incurable factors were $0 \%$ and 6 months, respectively, and these values in the 15 patients without incurable factors were $47.4 \%$ and 26 months $(P=0.035)$

patients was as follows: no evidence of disease, 3; alive with disease, 3; dead of other causes, 3; and dead of disease, 14. The overall 5-year survival rate was $34.3 \%$. The 5-year survival rate and the median survival of the 8 patients with incurable factors ( $\mathrm{pN} 3$, positive lavage cytology, and peritoneal dissemination) were $0 \%$ and 6 months respectively, while these values in the 15 patients without incurable factors were $47.4 \%$ and 26 months (Fig. 2). Four patients have survived for more than 5 years.

\section{Discussion}

In our data, of 195 patients with tumors invading the pancreatic head, 23 (12\%) underwent PD. This procedure has been rarely performed because of high morbidity and mortality rates. Prior to the 1990s, there had been only a few reports about this procedure [2-4]. Recently, with current advances in operative techniques, nutritional support, and antibiotics, some favorable results have been reported [5-11]. Ohashi [9] reported a large number of patients (145) undergoing this procedure. The 5-year survival rate of patients undergoing PD in that study was $6 \%$, and it was approximately equal to the result for patients undergoing more than $\mathrm{R} 0$ resection in our data. Thus, it is inferred that Ohashi's subjects included patients with far-advanced tumors that could not be removed by this procedure. With proper indications, PD could account for $10 \%$ of surgeries for tumors invading the pancreatic head, and the number of patients who would have this procedure would be around 30, even at a large institution.
In our study, tumor infiltration of the pancreatic head could not be confirmed in 7 patients $(30 \%)$ histopathologically. Such patients, theoretically, could have avoided this procedure; however, inconsistency between macroscopic and microscopic findings of infiltration has been reported to be $30 \%-50 \%$, often because of inflammatory reactions surrounding the tumor $[8,12]$. Even if the latest diagnostic modalities, such as computed tomography (CT), magnetic resonance imaging (MRI), and endoscopic ultrasound (EUS) are used, it is very difficult to distinguish between inflammatory reactions and tumor infiltration before operation. Intraoperative ultrasound could be more helpful than these modalities, but it was not used in any patients in the present series. It seems that inconsistency at a level of around $30 \%$ is unavoidable at present.

Morbidity after PD was in Ohashi's study [9] 51.6\% and $37.8 \%$ in that of Shchepotin et al. [11]. Regarding mortality, these authors reported rates of $6.3 \%$, and $10.8 \%$, respectively. Buchholtz et al. [1] recommended that PD should not be performed for gastric cancer because of an unacceptable risk, with no greater degree of palliation. The morbidity rate in our series $(73.9 \%)$ was higher than the rates in these previous reports $[9,11]$, to be sure. However, the operative mortality rate was $0 \%$ and all surviving patients could resume a regular life. Pancreatic fistula was the most common complication in this series. This is critical, as it may lead to intraabdominal abscess and rupture of arterial aneurysm. This complication was diagnosed by the detection of infectious drain discharge with a high concentration of amylase (>10000IU/1). For the early detection of pancreatic fistula, the concentration of amylase in the drain discharge is checked routinely after PD. When pancreatic fistula has developed, continuous drainage is performed, initially. If there is infection, continuous irrigation, using double-lumen drainage tubes, is done. To achieve better control of this complication, the medical staff including not only the surgeon but also nursing staff, have to be skilled at careful drain management. Therefore, this procedure should be performed only at institutions where PD for pancreatic cancer is frequently performed.

No patient in our series developed diabetes mellitus after PD, and only three required pancreatic exocrine enzyme support postoperatively. However, after PD with completion gastrectomy, two patients required TPN at home for a long period because of malnutrition. Total gastrectomy combined with PD should be considered very carefully, as nutritional problems may be severe.

The overall prognosis of patients with tumors invading the pancreatic head was poor; however the 5-year survival rate of patients undergoing $\mathrm{R} 0$ resection was about $30 \%$ in this series. In the patients with $\mathrm{R} 0$ resec- 
tions, there was no significant difference in survival between those requiring PD and those not requiring PD. Thus, to achieve R0 resection is an important objective, irrespective of whether or not PD is performed.

Ajisaka et al. [5] and Shchepotin et al. [11] reported that the 5-year survival rates of patients undergoing PD were $35 \%$ and $17 \%$, respectively. In a study of 26 patients undergoing PD combined with right hemicolectomy, Yonemura et al. [10] reported that the 5-year survival rate of 13 patients with tumors infiltrating the pancreatic head was $55 \%$. In our series, the 5-year survival rate for such patients was $34.3 \%$. In PD patients without incurable factors, the 5-year survival rate was higher, at $47 \%$, and 4 patients have survived for more than 5 years. Careful application of the PD procedure can achieve improved survival outcome. Kodama et al. [13] and Habu et al. [14] mentioned that a small amount of peritoneal dissemination and limited liver metastasis, respectively, were not contraindications for PD. However, most patients in the present series who had incurable factors died of the disease soon after operation. Incurable factors, such as $\mathrm{pN} 3$, positive lavage cytology, peritoneal dissemination, and visceral metastasis, should be regarded as a contraindication for PD.

In summary, the results after PD for patients with advanced gastric cancer with tumors invading the pancreatic head were acceptable from the aspects of morbidity, mortality, and survival benefit. If an $\mathrm{R} 0$ resection can be achieved by PD in such patients, this procedure should be performed. Patients with incurable factors should not be considered for PD. The combination of PD and total gastrectomy should be considered with caution.

Acknowledgments Dr. S. S. Mudan was supported by a grant from The Japanese Foundation for Cancer Research.

\section{References}

1. Buchholtz TW, Welch CE, Malt RA. Clinical correlates of resectability and survival in gastric carcinoma. Ann Surg 1978;188:7115 .
2. O’Brien PH, Mincey KH. Analysis of pancreatoduodenectomy. J Surg Oncol 1985;28:50-8.

3. Kishimoto H, Koga S. Evaluation of gastrectomy combined with the resection of other organs in the treatment of gastric cancer. Jpn J Surg 1979;9:173-9.

4. Scott HW Jr, Adkins RB Jr, Sawyers JL. Results of an aggressive surgical approach to gastric carcinoma during a 23-year period. Surgery 1985;97:55-9.

5. Ajisaka H, Fujita H, Kaji M, Maeda K, Yabushita K, Konishi K, et al. Treatment of patients with gastric cancer and duodenal invasion. Int Surg 2001;86:9-13.

6. Menjo M, Nimura Y, Hayakawa N, Kamiya J, Kondo S, Nagino $\mathrm{M}$, et al. Ten-year survival after pancreatoduodenectomy for advanced gastric cancer - report of two cases. Hepatogastroenterology 1999;46:1253-6.

7. Hirose K, Onchi H, Iida A, Katayama K, Yamaguchi A, Nakagawara G. Surgical results of pancreaticoduodenectomy for carcinoma of the distal third of the stomach. Int Surg 1999;84:1824.

8. Piso P, Bellin T, Aselmann H, Bektas H, Schlitt HJ, Klempnauer J. Results of combined gastrectomy and pancreatic resection in patients with advanced primary gastric carcinoma. Dig Surg 2002;19:281-5.

9. Ohashi I. Combined resection of adjacent organs for advanced cancer of the stomach: pancreatoduodenectomy and left upper abdominal evisceration (in Japanese). Surg Ther 1985;52:17380 .

10. Yonemura Y, Ooyama S, Matumoto H, Kamata T, Kimura H, Takegawa $\mathrm{S}$, et al. Pancreaticoduodenectomy in combination with right hemicolectomy for surgical treatment of advanced gastric carcinoma located in the lower half of the stomach. Int Surg 1991;76:226-9.

11. Shchepotin IB, Chorny VA, Nauta RJ, Shabahang M, Buras RR, Evans SR. Extended surgical resection in T4 gastric cancer. Am J Surg 1998;175:123-6.

12. Maehara Y, Oiwa H, Tomisaki S, Sakaguchi Y, Watanabe A, Anai $\mathrm{H}$, et al. Prognosis and surgical treatment of gastric cancer invading the pancreas. Oncology 2000;59:1-6.

13. Kodama I, Takamiya H, Mizutani K, Ohta J, Aoyagi K, Kofuji K, et al. Gastrectomy with combined resection of other organs for carcinoma of the stomach with invasion to adjacent organs: clinical efficacy in a retrospective study. J Am Coll Surg 1997;184:1622.

14. Habu H, Saito N, Sato Y, Takeshita K, Sunagawa M, Endo M. Results of surgery in patients with gastric cancer extending to the adjacent organs. Hepatogastroenterology 1990;37:417-20. 